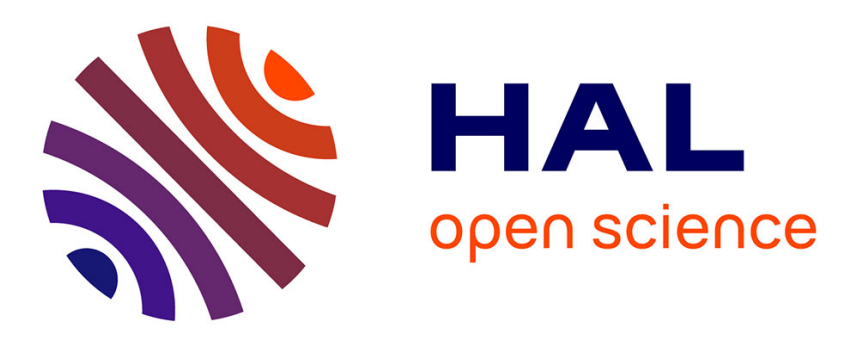

\title{
A comparison of several fault-tolerance methods for the detection and correction of floating-point errors in matrix-matrix multiplication
}

\author{
Valentin Le Fèvre, Thomas Herault, Julien Langou, Yves Robert
}

\section{- To cite this version:}

Valentin Le Fèvre, Thomas Herault, Julien Langou, Yves Robert. A comparison of several faulttolerance methods for the detection and correction of floating-point errors in matrix-matrix multiplication. [Research Report] RR-9351, Inria - Research Centre Grenoble - Rhône-Alpes. 2020. hal-02867859

\section{HAL Id: hal-02867859 \\ https://hal.inria.fr/hal-02867859}

Submitted on 15 Jun 2020

HAL is a multi-disciplinary open access archive for the deposit and dissemination of scientific research documents, whether they are published or not. The documents may come from teaching and research institutions in France or abroad, or from public or private research centers.
L'archive ouverte pluridisciplinaire HAL, est destinée au dépôt et à la diffusion de documents scientifiques de niveau recherche, publiés ou non, émanant des établissements d'enseignement et de recherche français ou étrangers, des laboratoires publics ou privés. 
A comparison of several fault-tolerance methods for the detection and correction of

floating-point errors in matrix-matrix multiplication

Valentin Le Fèvre, Thomas Herault Julien Langou Yves Robert

RESEARCH REPORT

$\mathbf{N}^{\circ} 9351$

June 2020

Project-Team ROMA 



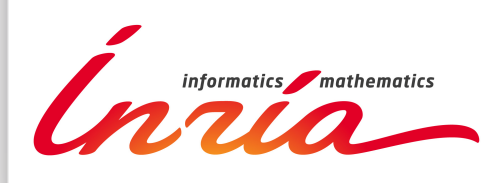

\title{
A comparison of several fault-tolerance methods for the detection and correction of floating-point errors in matrix-matrix multiplication
}

\author{
Valentin Le Fèvre*, Thomas Herault ${ }^{\dagger}$ Julien Langou ${ }^{\ddagger}$ Yves \\ Robert*† \\ Project-Team ROMA
}

Research Report n 9351 - June 2020 - 22 pages

\begin{abstract}
This report compares several fault-tolerance methods for the detection and correction of floating-point errors in matrix-matrix multiplication. These methods include replication, triplication, Algorithm-Based Fault Tolerance (ABFT) and residual checking (RC). Error correction for ABFT can be achieved either by recovering the corrupted entries from the correct data and the checksums by solving a small-size linear system of equations, or by recomputing corrupted coefficients. We show that both approaches can be used for RC. We provide a synthetic presentation of all methods before discussing their pros and cons. We have implemented all these methods with calls to optimized BLAS routines, and we provide performance data for a wide range of failure rates and matrix sizes. In addition, with respect to the literature, this paper consider relatively high error rates.
\end{abstract}

Key-words: Resilience, Matrix-matrix multiplication, Algorithm-Based Fault Tolerance (ABFT), Residual checking (RC), Silent errors.

${ }^{*}$ LIP, École Normale Supérieure de Lyon, CNRS \& Inria, France

† University of Tennessee Knoxville, USA

‡ University of Colorado Denver, CO, USA

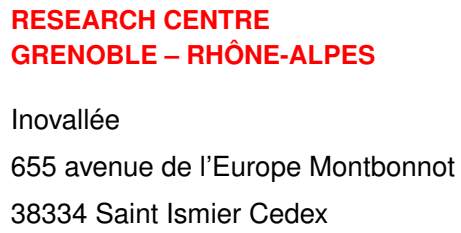




\section{Détection et correction des erreurs de calcul pour le produit de matrices (ABFT et Residual Checking)}

Résumé : Ce rapport compare plusieurs méthodes de détection et correction pour les erreurs de calcul dans le produit de matrices. Ces méthodes comprennent la réplication, ABFT (Algorithm-Based Fault Tolerance) et $\mathrm{RC}$ (Residual Checking). Pour ABFT et RC, la correction des erreurs peut s'effectuer soit par la résolution d'un système linéaire de petite taille, soit par le re-calcul des éléments corrompus. Nous présentons toutes ces méthodes de façon synthétique, avec leurs différences, leurs avantages et leurs inconvénients. Nous les avons toutes implantées et parallélisées avec des appels aux procédures BLAS natives,, et nous présentons des résultats de performances pour diverses tailles de matrices et différents taux d'erreurs.

Mots-clés : tolérance aux pannes, produit de matrices, Algorithm-Based Fault Tolerance (ABFT), Residual checking (RC). 


\section{Introduction}

Reliable computing has become a key challenge when deploying applications on large-scale platforms. These platforms are confronted to many errors striking during execution. These errors are due to the extremely large number of floating-point operations executed by the parallel applications that are deployed on such platforms. Indeed, the probability of facing a corrupted floating-point operation is proportional to the number of such operations that are executed $[5,11]$. Even if each processor exhibits a low individual error rate, the probability of several errors striking during the execution of the parallel application becomes very high with millions of cores running in parallel for a few days, or even hours.

There are very few ways to ensure that a whole application has executed without error. The only general-purpose method is to replicate the execution and to compare the results of both executions. If they do not coincide, an error has been detected, and the application must be executed a third time. To avoid a-posteriori re-execution, triplication can be enforced, which allows for error correction in addition to error detection, using a simple majority vote. However, triplication is even more costly than replication, which already requires half the resources to execute redundant operations.

Fortunately, many scientific applications heavily rely on scientific kernels from numerical linear libraries, and much of their floating-point operations are executed within these kernels. For most linear algebra kernels, application-specific methods have been devised for error detection and correction, with a much lower cost than replication. The most prominent application-specific approaches are Algorithm-Based Fault Tolerance (ABFT) and Residual Checking (RC), which we describe in full details in Section 2. Both ABFT and RC are known to enable error detection, but ABFT has received much more attention because it is also deployed for error correction. In theory, ABFT can correct up to $k$ errors with $2 k+1$ checksums $[16,19,20]$. However, the numerical instability of floating-point ABFT currently limits its usage to correct one or two errors within a kernel.

In this paper, we revisit the Residual Checking ( $\mathrm{RC}$ ) approach, and shows that it can be an efficient alternative to ABFT for error detection and correction. In particular, we focus on providing a transparent hardened version of some operation: the API, as exposed to the user, does not change, but the result is checked (and corrected if needed) before it is returned to the user. This creates a problem for ABFT, as the efficiency of the technique lies in mixing the user data and the redundant data used for failure detection and correction (see Section 2.2). RC can be implemented without modifying the API of the original computation kernel (see Section 2.3), which is a key advantage from a software engineering perspective.

Another drawback of ABFT compared to RC is the lack of flexibility. By construction, ABFT uses a fixed number of checksums chosen a priori, say 
$2 k+1$, and will fail if more errors than $k$ errors strike during the kernel. On the contrary, $\mathrm{RC}$ adapts the number of verifications on the fly, as a function of the number of errors found.

We adopt a somewhat narrow focus and only deal with protecting matrixmatrix multiplication from floating-point errors. Matrix-matrix multiplication is the archetypal linear kernel and is at the heart of several linear solvers, hence it is one of the most important kernels to study. Assessing the efficiency of residual checking for matrix-matrix multiplication will lay the foundations for the study of a full dense linear algebra library. The major contributions of this paper are the following:

- A synthetic comparison of several fault-tolerance methods for error detection and correction in matrix-matrix multiplication, including novel approaches for RC

- A publicly-available prototype implementation of all the methods, with calls to optimized BLAS kernels

- A comparative assessment for a wide range of failure rates and matrix sizes.

The paper is organized as follows. We review existing fault-tolerant approaches work in Section 2, covering replication, ABFT and RC. Section 3 is devoted to related work, and builds upon the classification introduced in Section 2. Section 4 is the heart of the paper: we describe our publiclyavailable implementations and provide a detailed experimental comparison of all methods. Section 3 is devoted to related work. Finally, we conclude and give hints for future work in Section 5 .

\section{Methods}

This section provides an overview of replication (Section 2.1), ABFT (Section 2.2) and RC (Section 2.3), and concludes with a detailed comparison of ABFT and RC (Section 2.4).

\subsection{Replication}

The first approach to detect computational errors is also the only systemic approach that can apply to any algorithm: it consists in replicating computations, and checking that both executions produce the same result. In the context of mutable data, this also implies to work on a copy of the data to compute, in order to enable the replicated computation $[14,15,2]$. There are multiple ways to implement replication: the computations can be executed sequentially, one after the other, at any level of granularity, or in parallel. Ultimately, the replication process provides two copies of the output of the computation and these copies are compared bit-to-bit, to detect errors. 
Any error detected can then be resolved with a voting process: more replicas are computed, and if (at least) two output results converge on a same result, this result is considered valid. The probability that two computation errors produce the same result is considered negligible, since errors are supposed to be independent and identically distributed random variables.

\section{$2.2 \quad$ ABFT}

Algorithm Based Fault Tolerance (ABFT) is an approach introduced in [13], that leverages mathematical properties of the algorithm to introduce redundancy in the data and thus allows to detect, and sometimes locate and correct errors during a computation. Applied to the matrix-matrix multiplication of the $C \leftarrow A B$ as an example, where $A$ is $n$-by- $n$ and $B$ is $n$-by- $n$, the main idea of ABFT is to extend the matrix on which the operation is applied with checksum vectors that are pre-computed before the matrix-matrix multiplication. This gives

$$
\begin{gathered}
A \text { is extended with }\left(\begin{array}{c}
A \\
A_{c}
\end{array}\right) \text { with } A_{c}=v^{T} A \\
B \text { is extended with }\left(\begin{array}{cc}
B & B_{r}
\end{array}\right) \text { with } B_{r}=B w
\end{gathered}
$$

where $w$ and $v$ are checksum generator vectors. Once $A$ and $B$ have been augmented, we perform the matrix-matrix multiplication

$$
\left(\begin{array}{cc}
C & C^{(r)} \\
C^{(c)} & C^{(\alpha)}
\end{array}\right) \leftarrow\left(\begin{array}{c}
A \\
A^{(c)}
\end{array}\right)\left(\begin{array}{ll}
B & B^{(r)}
\end{array}\right)
$$

and we see that we must have the following relations

$$
C^{(r)}=C w \quad \text { and } \quad C^{(c)}=v^{T} C \quad \text { and } \quad C^{(\alpha)}=v^{T} C w .
$$

Therefore, a way to check that the entries of $C$ have been correctly computed is to check that the equalities in Equation (1) hold. With this scheme, we can, for example, guarantee to detect any single error in $C$. (In other words, if no more than one entry of $C$ is corrupted, then this scheme will detect the error.) Now we can also observe that

1. $w$ and $v$ does not have to be vectors, but they can also be block of vectors,

2. The whole realm of error correction codes (e.g. Reed Solomon error correction code) is now at our doorstep since for each row $C_{i}$ of $C$, we have computed $C_{i}$ and its checksum with respect to $w, C_{i} w$, and so not only can we detect errors, but we can also locate and recover errors. Using Reed Solomon error correction code, for example, we can detect, locate, and recover $k$ errors with $2 k+1$ checksums. (Provided that we use an appropriate encoding block of vectors $w$.) 
3. The Reed Solomon algorithm is notoriously unstable in finite precision arithmetic [8] and does not enable to recover from many errors or to handle very long vectors.

4. For detection, in practice, one row checksum of the form $C_{i} w$ is often enough to detect errors in any row of $C, C_{i}$. We simply check whether $C_{i} w=C_{i}^{(r)}$. This check can fail if the error vector introduced in $C$ is orthogonal to $w$. However this is unlikely.

5. Tolerance of the order of machine precision has to be added to the check. Indeed, we only attend to detect errors that are larger than the errors made by the round-off errors of the numerical computation. So we check, for example,

$$
\left\|C^{(r)}-C w\right\|_{2} \leq 10 u\|A\|_{\text {fro }}\|B\|_{\text {fro }}\|w\|_{\text {fro }}
$$

where $u$ is the machine roundoff and the number " 10 " is taken arbitrarily. (Current numerical error theory [12] has established that "10" is, with high probability, the function $\sqrt{n \log (n)}$ where $n$ is the largest dimension in the matrix-matrix multiplication.)

6. A standard way to locate errors is to use "coordinate checkpointing" [22, 21]. So if the row checksum $C_{i}^{(r)}$ is not $C_{i} w$ and the column checksum $C_{j}^{(c)}$ is not $v^{T} C_{j}$ then we conclude that the entry $c_{i j}$ is false.

7. Once an error is located, this is done, we can either recover the $c_{i j}$ through the redundancy introduced by the checksum and therefore solving a system of linear equations with unknown $c_{i j}$, this leads to the method ABFT-SOLVE, or we can, in the case of matrix-matrix multiplication, simply recompute the entry $c_{i j}$ from the $i$ th row of $A$ and the $j$ th row of $B$, this leads to the method ABFT-RECOMP.

8. We note that one advantage of Reed Solomon is that it enables to locate and correct with checksum only on the rows or only the columns. Coordinate checkpointing would need both row and column checksums. For matrix-matrix multiplication, it is convenient to maintain both checksums, while for other linear algebra operations, this is not always natural.

9. How to choose $v$ and $w$ ? In the case ABFT-solve, Chen and Dongarra $[7,8]$ showed that taking random matrices enable to recover the solution with high probability during the linear solve to recover the corrupted entries. While less critical, it does seem a good idea to also take random vectors $v$ and $w$ for ABFT-RECOMP.

10. As for the overhead, we see that to encode and compute with $k$ checksums with $k \ll n$ is $\mathcal{O}\left(n^{3}\right)$ flops, the cost to detect, locate and recover $\ell$ 
errors is $\mathcal{O}\left(n^{2} \ell\right)$ flops. Therefore the cost (in term of flops) of recovery is theoretically negligible compared to the cost of computation.

11. We consider a square matrix-matrix multiplication, but the rectangular case is similar.

12. This paper is concerned with soft errors also called silent errors. In this case, (1) we need to detect errors, (is the computed data correct or not?,) (2) we need to located errors, (if the data is not correct, which entries are corrupted?,) and (3) we need to correct errors. Another interesting and related problem [6,3], but much easier, is when failure (also called fail-stop) happens. In fail-stop case, we know that there are errors, we know where the errors are, and so we are left to only correct errors.

Altogether, there are various layers and possibilities on how to use ABFT. We describe a similar technique, Residual Checking (RC) in Section 2.3 before coming back to ABFT and discussing the differences with RC.

\subsection{Residual Checking ( $\mathrm{RC})$}

A closely related method is $\mathrm{RC}$, which exploits the fact that checking the correctness of the result of a computation is usually easier than computing it. In short, one more time using the $C \leftarrow A B$ matrix-matrix multiplication as an example, if one wants to check at low cost whether $C$ is correctly computed, one can compute, on the one hand, $C w$ and, on the other hand, $A(B w)$ and check whether these two vectors are similar. And, not surprisingly, the two methods ABFT and RC share similar characteristics: (1) Low cost, (2) if $w$ is in the nullspace of $C-A B$, the error matrix, then we will not detect the errors, however this is unlikely, etc. As one can see $\mathrm{RC}$ is very similar to ABFT. And actually the difference is not clear. Historically RC was introduced with "error detection" in mind only. So you would perform the computation, use $\mathrm{RC}$ to detect errors, and then redo the computation if any error is detected. Examples of such applications of RC are matrix-matrix multiplication and QR factorization [17], and the Eigenproblem [18]

We want to correct a long held misconception about RC. RC has long be thought to only be able to detect errors, and not able to locate and correct errors. For example, Prata and Silva [17] writes: "We left out of our comparison one aspect where ABFT would do better than $R C$, namely fault localization and error recovery, ( $R C$ has no such capability)." Actually, in very much the same way as $\mathrm{ABFT}, \mathrm{RC}$ is able to detect, locate and correct errors. The two methods (ABFT and RC) are essentially similar and have the same capabilities. 


\subsection{Differences between ABFT and RC}

There is a fundamental principle difference between RC and ABFT.

Given some input, an algorithm computes some output such that a relation is true. For example, given $A$, (1) LU factorization: compute $P$, $L$, and $U$ such that $P A=L U$, (2) QR factorization: compute $Q, R$ such that $A=Q R$, (3) SVD decomposition: compute $U, \Sigma$, and $V^{T}$ such that $A=U \Sigma V^{T}$. RC finds a quick way to check whether this final relation holds. For example, given a vector $x,(1)$ check that $P(A x)=L(U x)$, (2) check that $A x=Q(R x),(3)$ check that $A x=U\left(\Sigma\left(V^{T} x\right)\right)$. If the relation does not hold, then $\mathrm{RC}$ has succeeded in detecting an error. If the relation holds, then RC has succeeded in assessing (with high probability) the correctness of the result.

On the contrary, ABFT starts with checksums on the initial data, and maintains the consistency of the checksums along the algorithm. So the checksums are being modified as the data is being modified so that current data is consistent with current checksum.

As a side comment, the difference above explains why it is easier to derive $\mathrm{RC}$ for many more algorithms than for ABFT. (In a few lines, we gave $\mathrm{RC}$ for three algorithms, and for ABFT, we barely explained how ABFT works and we certainly did not give concrete information on a specific implementation.) However, in the case of matrix-matrix multiplication and linear algebra in general, once $\mathrm{RC}$ and $\mathrm{ABFT}$ algorithms have been implemented, the differences between $\mathrm{RC}$ and $\mathrm{ABFT}$ are not so clear any longer, and we find that the algorithms are often very close. We describe the design space as having three dimensions. These three dimensions are essentially orthogonal in the sense that it is possible to make choices in any dimension independently of the others.

Dimension 1: appending checksums or leaving checksums separate. The checksums (for example $A_{c}$ ) can either (case 1ab) be appended to the main matrix (e.g. as extra rows to $A$ ) or (case 1rc) left as separate independent blocks of vectors. Discussion:

1. On the one hand, for RC, the checksums are naturally separate from the matrices. On the other hand, ABFT has been presented with both possibilities. RC is always $1 \mathrm{ab}$. ABFT can be $1 \mathrm{ab}$ (e.g., [3, 13]) or $1 \mathrm{rc}$ (e.g., $[21])$.

2. One advantage of leaving the checksums separate from the matrices is to not change the data structures of the original (non fault-tolerant) code. This is much easier to accomplish from a software engineering point of view.

3. One advantage of appending the checksum is to call kernels only once (on the extended data structure). The computation on the checksums 
is then processed at the same time as the computation on the main matrix. This can be much faster.

Dimension 2: computing checksums on input data before computation or after. If we compute the initial checksums before the matrixmatrix multiplication, we call this 2ab. If we compute the initial checksums after the matrix-matrix multiplication, we call this 2rc. Discussion:

1. The main distinction between $2 \mathrm{ab}$ and $2 \mathrm{rc}$ is not really when we compute checksums, but more whether we "can" recompute initial checksums after the main operation. Recomputing the initial checksums after the computation means that we are storing the input data, and we are not overwriting in the initial data with computation. In $\mathrm{Nu}-$ merical Linear Algebra, this is a significant constraints since we often have one operand that is in/out. If we perform $2 \mathrm{rc}$, we must use backup (copy) of all in-out operands.

2. It seems that, in the literature, ABFT always compute the initial checksums before the computation.

3. If one wants to append the checksums to the matrix, then one will in general compute the checksums before the computation. Therefore, often, $1 \mathrm{ab} \Rightarrow 2 \mathrm{ab}$. (And its contrapositive: $2 \mathrm{rc} \Rightarrow 1 \mathrm{rc}$ : if we compute the checksums after, then the checksums will be separate.)

4. One advantage to compute the checksums after is to compute as many initial checksums as needed by the number of errors, this is particularly useful to lower the overhead, and to avoid making any assumption on the maximum number of errors that will be encountered.

5. Because the recovery step of ABFT is numerically not guaranteed to work, ABFT schemes often backup (copy in temporary buffer) input data, or they combine ABFT with checkpointing [9]. In short, if ABFT detects an error, it attempts to recover the corrupted entries from the checksum, if some numerical instabilities are detected after the recovery, the whole computation is done again (akin to replication). Most of the time, there are no errors. When an error occurs, a fast recovery from the checksum is attempted. If this fails, the whole computation is redone. Without these backups, we cannot guaranteed an ABFT code to succeed. Because these backups are necessary for reliability and often used, it is often the case that the input matrix are available at the end of a computation in ABFT, and, in practical implementation of ABFT, we can compute checksums (2rc) after the computation. 
Dimension 3: detect+recompute or detect+locate+lazy-recompute or detect+locate+solve. Case 3rc: detect errors, and recompute the whole computation if some errors are detected 3rc. Case 31o: detect errors, locate errors and recompute only the corrupted entries (also called lazy recomputation in [21].) Case 3ab: detect errors, locate errors and recover the corrupted entries from the redundant information in the checksum, we call this 3ab. Discussion:

1. a long-held misconception is that computing initial checksum after does not enable to recover corrupted entries from the checksum. In other words the misconception is $2 \mathrm{rc} \Rightarrow 3 \mathrm{rc}$. As already explained this is false.

2. For 310 and $3 a b$, in this paper, the localization is done through "coordinate checkpointing".

3. 310 assumes that entries can be recomputed somewhat easily from only the input data, and maybe some non-corrupted entries. It is not obvious that there are many kernels for which this is possible. Matrixmatrix multiplications is one such kernel.

4. For 3ab, assuming that we can locate the errors, (through coordinate checkpointing, for example,) Chen and Dongarra [7,8] showed that taking random matrices enable to recover the solution with high probability during the linear solve to recover the corrupted entries.

5. Reed-Solomon encoding enables $3 a b$ with either a row checksum or a column checksum, it does not require both row and column checksum. This is very useful for some operations. (Not matrix-matrix mutiplication though.) However the checksum block of vectors $v$ and $w$ are extremely ill-conditioned and leads to numerically unstable codes.

6 . we note that $2 a b+3 a b$ is the only way (in this design space) to overwrite in/out operands during the computation and recover from errors. All other methods needs to copy and store in/out operands to extra memory space to be able to recompute from the input in case an error occurs.

Which dimension distinguishes ABFT vs RC. Dimension 1: we can distinguish $\mathrm{ABFT}$ and $\mathrm{RC}$ by defining $\mathrm{ABFT}$ as appending checksums to matrices, and RC as having checksum separate from matrices. Dimension 2: we can distinguish $\mathrm{ABFT}$ and $\mathrm{RC}$ by defining $\mathrm{ABFT}$ as computing the initial checksums before computation, and RC as computing the initial checksums after computation. Dimension 3: we can distinguish ABFT and RC by defining $\mathrm{RC}$ as detecting and maybe locating errors, and following a detection by recomputation, and defining $\mathrm{ABFT}$ as recovering the corrupted entries, 


\begin{tabular}{|c|c|c|c|c|c|c|c|}
\hline Reference & $1 \mathrm{ab}$ & $2 \mathrm{ab}$ & $3 \mathrm{ab}$ & $1 \mathrm{rc}$ & $2 \mathrm{rc}$ & $3 \mathrm{rc}$ & 310 \\
\hline$[13]$ & $\checkmark$ & $\checkmark$ & $\checkmark$ & & & & \\
\hline$[17]$ & & & & $\checkmark$ & $\checkmark$ & $\checkmark$ & \\
\hline$[10]$ & & & & $\checkmark$ & $\checkmark$ & $\checkmark$ & \\
\hline$[6]^{*}$ & $\checkmark$ & $\checkmark$ & $\checkmark$ & & & & \\
\hline$[3]^{*}$ & $\checkmark$ & $\checkmark$ & $\checkmark$ & & & & \\
\hline$[1]$ & & & $\checkmark$ & $\checkmark$ & $\checkmark$ & & $\checkmark$ \\
\hline$[21]$ & & $\checkmark$ & & $\checkmark$ & & & \\
\hline
\end{tabular}

Table 1: Taxonomy of related work

after detection and location, from the redundant information contained in the checksum. In our mind, (1) the two methods ABFT and RC are very close and it might be futile to attempt to differentiate them, (2) we have explained that the problem has at least 3 dimensions, and a taxonomy in 1 dimension (ABFT vs RC) is too coarse and makes things confusing.

Another consideration: when only either row or column checksums are possible. This paper considers matrix-matrix multiplication where row and column checksums are both possible. However quite a few operations only enable one side for the checksum. We can either have a row checksum or a column checksum but not both. In this case, we can detect errors and redo computation. As far as localizing errors, since we cannot do coordinate checkpointing, we need to use some kind of Reed Solomon code to locate errors. This study is beyond the scope of this paper.

\section{Related work}

Multitudinous papers have been published on replication, ABFT and RC. Recent surveys on ABFT are provided in $[4,9]$. We have selected below a small set of closely related works, which we classify in Table 1 according to the criteria given in Section 2.

Among these works, [1] is the first paper that we know of that use a residual-checking-like with a "solve" (as opposed to recompute the entries). [21] is the first to introduce the strategy to detect, locate and recompute only what is corrupted. We note that the authors decide against $3 a b$ because they "have concerns that subtracting the estimated error from the computed result may give rise to numerical stability issues, mainly due to catastrophic cancellation." In the case of matrix-matrix multiplication where the entries of $C$ can be independently computed, we share this point of view. Also the paper introduces the idea of "lazy left checksums", in short, compute 
column checksums (called right checksums in [21]) to detect errors, if some errors are found, then compute the row checksums (called left checksum) so as to locate. Note that their approach involves partitioning the original matrices into blocks of appropriate size and to apply protection techniques at the block level, restricting to environments with low fault-rates, so that they basically detect and correct at most one error per block product.

\section{Experiments}

\subsection{Implementations}

We implemented variants of all the techniques discussed above. The implementation is in C, relying on the BLAS kernels for all linear algebra operations (namely GEMM and GEMV), and each hardened routine provides the same API as the GEMM routine defined by BLAS, but implements a different error detection and correction strategy. Here is the list of the six routines that we implemented, and that we compare in Section 4.3:

- NoFT is only used as a reference point, and is a direct call to the GEMM routine provided by the BLAS library, without any error checking nor correction strategy.

- Replication uses the most simple (and systematic approach): replication, as described in Section 2.1: the GEMM operation is computed twice, then resulting elements are compared one by one, and if an error is detected, the entire operation is computed a third time. Elements are then selected by a simple majority vote, and if no majority can be obtained for some element, the operation is applied again, until a pair of matching results can be found.

- ABFT-Solve $(=1 \mathrm{ab}+2 \mathrm{ab}+3 \mathrm{ab})$ is the traditional ABFT method: the input matrices are copied into larger matrices, that are extensions of the inputs with a fixed number of column and row checksums. These checksums are computed from the initial data, and the GEMM operation is applied on the extended matrix. After it completes, we check the checksums to detect errors. If errors are detected, a linear system of equations is solved as described in $[3,6,16,19,20]$ to compute the corrected values, and the resulting matrix is copied in the output parameter.

- ABFT-REComp (=1ab +2ab +3lo) follows the same strategy as ABFTSOLVE to detect errors, but the matrix is extended with a single column and row as checksums. By crossing the columns in which the rowchecksum is incorrect and the rows in which the column-checksum is 
incorrect, we extract a number of suspected wrong results, and we recompute only these elements from the input data. The result is checked (iterating another step of re-computation if needed), and copied back into the output parameter.

- RC-SOLVE $(=1 \mathrm{rc}+2 \mathrm{rc}+3 \mathrm{ab})$ uses the residual checking approach to compute the checksums (see Section 2.3): the GEMM operation is computed, and once it is computed, a single column checksum is generated randomly, and the routine compares how applying the output of GEMM on it differs from applying the two input matrices. If the result differs in any element, there is at least an error on the corresponding row(s). Additional checksums are then generated, until a system of linearly independent equations can be formed. That system is solved to correct the errors.

- RC-RECOMP $(=1 \mathrm{rc}+2 \mathrm{rc}+310)$ uses the same approach as RCSOLVE, until the correction phase is reached. When this is the case (there is at least one row with errors), a row-checksum is computed (as the column checksum was), and by crossing the row-checksum errors and the column-checksum errors, we can approximately locate suspected error locations. These elements of the output matrix are recomputed from the initial data to patch the result matrix which is returned by the routine.

\subsection{Setup}

For introducing errors in the operations, we use a parameter $r$ which is the error rate of one floating-point operation. We compute the probability for an element to be erroneous, knowing it is the result of $m$ operations: $P=1-(1-r)^{m}$ and we modify each element that has been drawn to be corrupted by randomizing the element. We first apply this modification on all the elements of the matrix after the GEMM operation, with $m=2 n-1$, because there are $n$ multiplications and $n-1$ additions per element when multiplying square matrices of size $n$. Then, for the recomputed elements of RC-RECOMP and ABFT-RECOMP implementations, we set $m=2 n-1$ for each element that is recomputed from scratch and we check again the result. For RC-SOlve and ABFT-Solve, $m=c^{2}$ where $c$ is the number of corrupted columns in the matrix. Finally for REPLicAtion, $m=2 n-1$ for each element of every new matrix computed. Tables 2 and 3 detail the average number of errors in the matrix after the first GEMM operation (column Initial) and the average number of errors that appear during the (multiple) correction(s) (column Correction) for $N=1000$ (Table 2) and $N=3000$ (Table 3). In each experiment, the maximum duration of the hardened operation is bounded by 4 iterations of the applied check / correct procedure, and if the matrix is still corrupted at this point, the operation 
Detection and correction of floating-point errors in matrix-matrix multiplication 14

\begin{tabular}{|l|c|c|c|c|c|c|}
\hline \multicolumn{1}{|c|}{ Rate } & \multicolumn{2}{c|}{$10^{-10}$} & \multicolumn{2}{c|}{$10^{-9}$} & \multicolumn{2}{c|}{$10^{-8}$} \\
\hline Location & Initial & Correction & Initial & Correction & Initial & Correction \\
\hline NoFT & 0.13 & - & 1.91 & - & 19.43 & - \\
\hline ABFT-RECOMP & 0.20 & 0 & 2.13 & 0 & 19.84 & 0.02 \\
\hline ABFT-SOLVE & 0.18 & 0 & 2.00 & 0 & 19.75 & 0 \\
\hline RC-RECOMP & 0.24 & 0 & 1.97 & 0 & 19.68 & 0.02 \\
\hline RC-SOLVE & 0.19 & 0 & 2.00 & 0 & 20.64 & 0 \\
\hline REPLICATION & 0.14 & 0.21 & 2.10 & 4.02 & 20.39 & 41.44 \\
\hline
\end{tabular}

Table 2: Average number of erroneous elements in the matrices of size $N=1000$ : Initial counts the number of errors after the first GEMM; Correction counts the number of errors during the correction phase.

\begin{tabular}{|l|c|c|c|c|c|c|}
\hline Rate & \multicolumn{2}{|c|}{$10^{-10}$} & \multicolumn{2}{c|}{$10^{-9}$} & \multicolumn{2}{c|}{$10^{-8}$} \\
\hline Location & Initial & Correction & Initial & Correction & Initial & Correction \\
\hline NoFT & 5.43 & - & 52.75 & - & 534.19 & - \\
\hline ABFT-RECOMP & 5.37 & 0 & 54.03 & 0.04 & 541.42 & 14.79 \\
\hline ABFT-SOLVE & 5.38 & 0 & 55.43 & 0.02 & 541.85 & 794.42 \\
\hline RC-RECOMP & 5.66 & 0 & 54.04 & 0.01 & 539.77 & 15.15 \\
\hline RC-SOLVE & 5.54 & 0 & 53.12 & 0 & 539.51 & 811.35 \\
\hline REPLICATION & 5.26 & 10.49 & 54.73 & 110.64 & 543.58 & 1149.37 \\
\hline
\end{tabular}

Table 3: Average number of erroneous elements in the matrices of size $N=3000$ : Initial counts the number of errors after the first GEMM; Correction counts the number of errors during the correction phase.

is considered failed. ABFT-SOLVE needs one additional parameter which is the number of checksums to add to the matrix: we set it to $2 \times 2 N^{3} r$ as $2 N^{3} r$ is the expected number of failures during the computation and we want a margin to tolerate more errors in bad scenarios. If ABFT-SOLVE cannot solve the system of equations, the operation is considered as failed.

We run the experiments with 16 cores out of a 20-core Intel Xeon CPU E5-2650 v3 at $2.30 \mathrm{GHz}$, with $64 \mathrm{~GB}$ of memory hosted at the University of Tennessee. The code is compiled with GCC 9.2.0, and the BLAS kernels where provided by Intel MKL version 2019.3.199. We evaluate both the sequential and multi-threaded versions of the algorithms. We run 100 iterations of each combination of implementations and parameters (the matrix size $N$ and the error rate $r$ ) and we average the execution times of the different parts of the algorithm. DGEMM is the time spent doing the main operation (and subsequent DGEMMs for REPLICATION); Check is the time spent computing the checksums and finding the location of the errors; Correct is the time spent recomputing or solving the systems depending on the chosen implementation. We report the execution times when each of the 

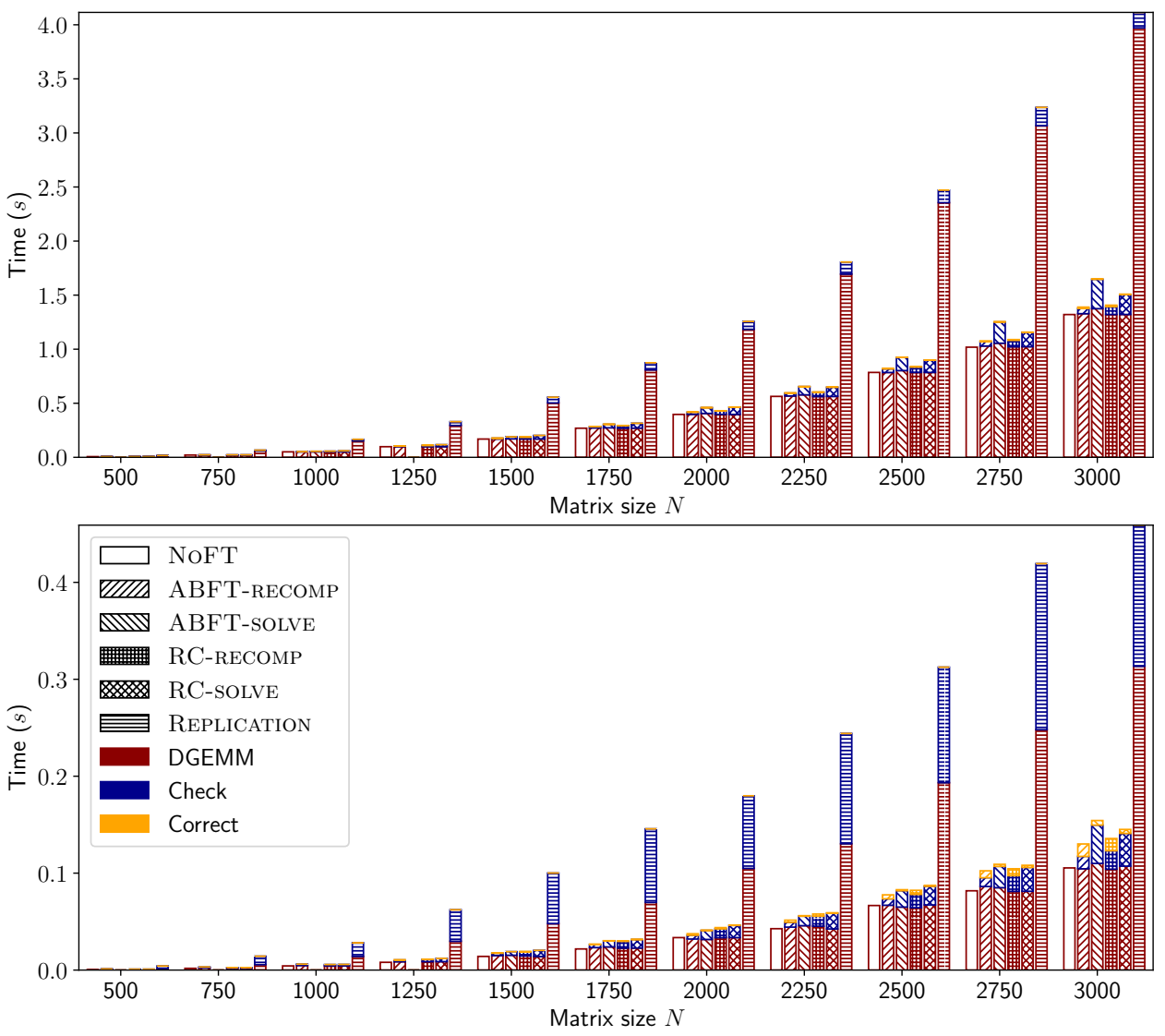

Figure 1: Sequential (top) and multi-threaded (bottom) algorithms for an error rate of $10^{-9}$.

100 iterations succeeds; otherwise, we report the number of failed iterations. As a reference, we show the time to execute a GEMM on a $N \times N$ matrix without fault tolerance nor failure injection under the name NoFT. The source code of the implementations used for the experiments is available at https://github.com/vlefevre/abft-rescheck.

\subsection{Results}

Figure 1 describes the detailed execution of our 6 implementations for an error rate $r=10^{-9}$ and a varying matrix size $N$. The first thing to notice is that replication is always the less efficient technique. Indeed, even without failures, two full DGEMM operations need to be executed to detect failures. Moreover, every time there is at least one error during the computation, we need to compute the resulting matrix a third times to correct it. It is enough to correct in most cases but the cost of a DGEMM operation, especially in sequential, is much bigger than the cost of a detection and the 
Detection and correction of floating-point errors in matrix-matrix multiplication 16

\begin{tabular}{|l||c|c|c|c|c|c|c|c|c|}
\hline \multicolumn{1}{|l||}{ Implementation } & \multicolumn{7}{|c|}{ ABFT-SOLVE } & \multicolumn{2}{c|}{ RC-SOLVE } \\
\hline \hline Error rate $r$ & $10^{-10}$ & \multicolumn{1}{|c|}{$10^{-9}$} & $8 \times 10^{-9}$ & $10^{-8}$ & $8 \times 10^{-9}$ & $10^{-8}$ \\
\hline Matrix size $N$ & 3000 & 500 & 750 & 1000 & 1250 & 3000 & 3000 & 3000 & 3000 \\
\hline Sequential & 4 & 2 & 23 & 0 & 7 & 1 & 3 & 11 & 78 \\
\hline Multi-threaded & 3 & 2 & 24 & 4 & 3 & 0 & 4 & 15 & 81 \\
\hline
\end{tabular}

Table 4: Number of failed iterations (over 100) for the parameters used in Figures 1 and 2 .

ensuing correction at this error rate.

The overheads of detecting and correcting errors for all methods but REPLICATION remain small, even when the matrix size (thus the number of errors) increases: there is only a small proportion of the output matrix that is corrupted, and thus the amount of recomputation or the size of the linear problem to solve to correct are small. Recomputation-based approaches, however, outperform significantly system-solving approaches.

The multi-threaded case shows the same characteristics overall, except the check time of REPLICATION is significantly increased, relative to the duration of the GEMMs. As checking for REPLICATION is a memory-bound problem, when all the cores access the memory simultaneously, the memory bus becomes the bottleneck and limit parallel efficiency.

When $N$ increases, both RC-SOLVE and ABFT-SOLVE are likely not to correct everything within 4 re-executions as the correction is done by solving linear systems of size c, hence with $O\left(\mathrm{c}^{3}\right)$ flops, where c is the number of corrupted columns. For a given error rate, increasing $N$ will increase both the number of columns and the probability that it is corrupted at the beginning. Thus the number of operations involved in the solve phase $\left(c^{2}\right.$ compared to $2 n-1$ ) can quickly grow and we need more iterations to finish. ABFT-SOLVE also does not always correct for small error rates or small matrix sizes (see Table 4). As the margin on the number of checksums to add is smaller, it becomes easy to have more errors than what we estimated even if we already added a factor 2 to the expected number of failed operations. This risk is managed by the RC-SOLVE implementation as the checksums are computed after failures hit the initial DGEMM operation, and thus the exact minimal number of checksums is used.

Figure 2 shows the same measurements, but with a fixed problem size $(N=3000)$ and a varying error rate. The Solve-based approaches do not produce results at $8 \times 10^{-9}$ and $10^{-8}$ error rates in the sequential case, and ABFT-SOLVE only produce an output in a very long time in the multithreaded case with an error rate of $8 \times 10^{-9}$. As the number of columns including errors gets closer to $N$, the size of the system to solve becomes closer to the size of the original matrix. Since errors can also impact these computations, with a higher probability, the solve-based approaches fail, 

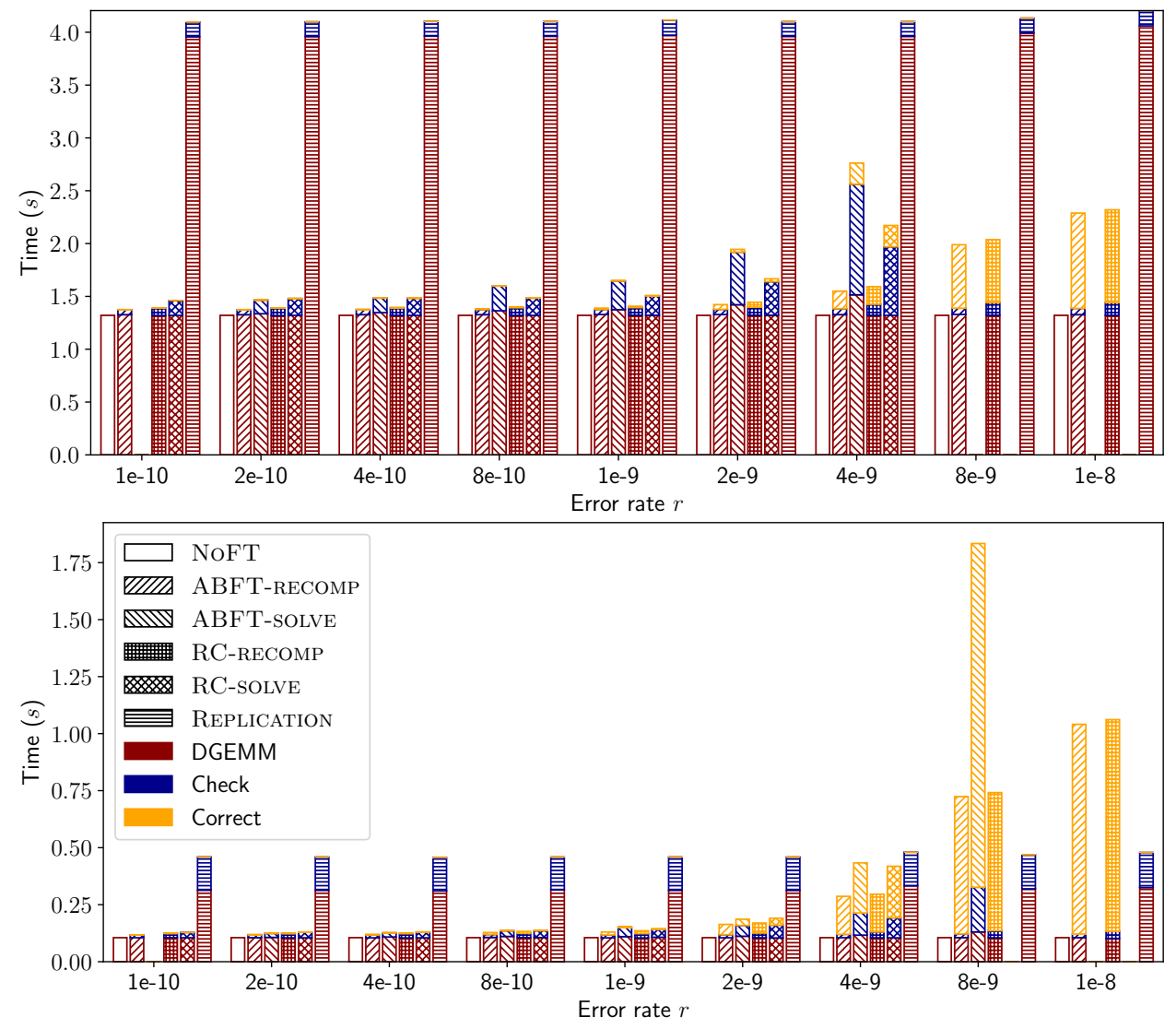

Figure 2: Sequential (top) and multi-threaded (bottom) algorithms for a matrix size of 3000 . 

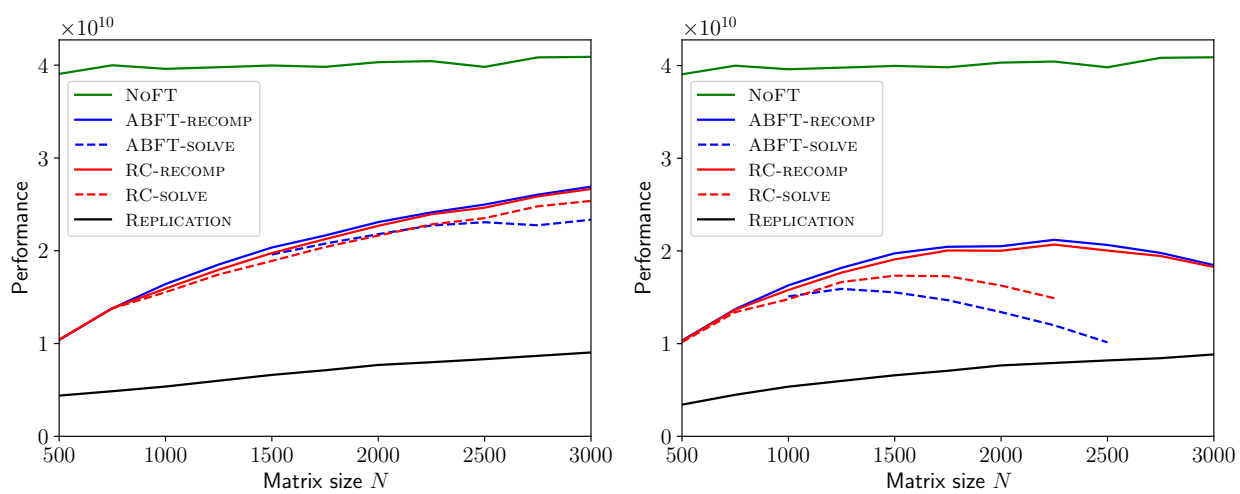

Figure 3: Overall performance of the 6 algorithms for $r=10^{-9}$ (left) and $r=10^{-8}$ (right).

leading to repeated iterations of the correction process.

For low error rates, RC-RECOMP and ABFT-RECOMP are the two best performing algorithms and behave very similarly. The main difference between the two algorithms is that RC-RECOMP is easier to (1) set up since the check is done after the main computation and does not depend on the algorithm (for detection) and (2) to use as a blackbox for the user with no conversion of data needed. This last point is important as a user-friendly library would take as input $N \times N$ matrices and ABFT needs to add some extra steps to compute a bigger matrix with the checksums in it. This can quickly increase the execution time (and the memory footprint) of the algorithm if only a few DGEMM operations are done in a row because of the memory allocations and copies.

However, as the error rate increases, the recomputation-based approaches start to show slower corrections. This is particularly visible in the multithreaded case: REPLICATION eventually outperforms RC-RECOMP and ABFTRECOMP. This can be explained by two things: first, REPLICATION's efficiency is independent from the error rate, because errors hit independent elements in the 3 computed matrices; second, as the number of errors in the matrix gets closer to $N^{2}$, the recomputation algorithm is less efficient than re-doing a fully optimized GEMM: it implements a parallel loop over the failed elements of sequential dot products. In the multi-threaded case, this is less efficient than re-computing the entire GEMM.

Finally, we sum up these results in Figures 3 and 4 . We represent here the performance of the operations, as the ratio between $2 N^{3}$ (the number of floating point operations in a GEMM) and the execution time of the sequential algorithms. It is clearly visible that the error rate has no influence on REPLICATION while ABFT-RECOMP and RC-RECOMP are the two best performing algorithms and their performance is equivalent. We also see that their performance stays close to that of NoFT as long as both $r$ and $N$ do 

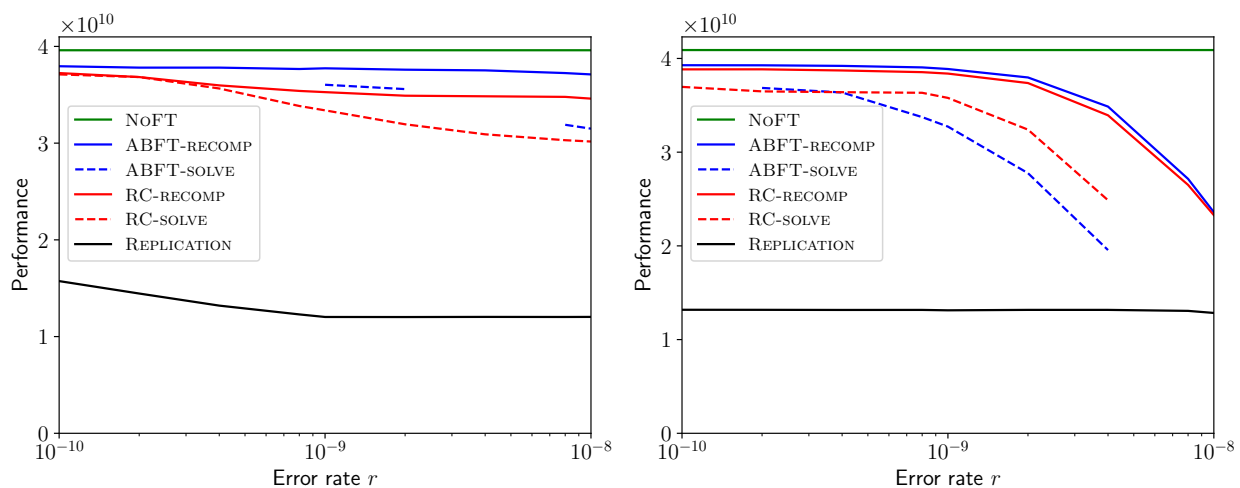

Figure 4: Overall performance of the 6 algorithms for $N=1000$ (left) and $N=3000$ (right).

not become too big.

\section{Conclusion}

In this paper, we have reviewed and compared ABFT and Residual Checking $(\mathrm{RC})$ for detecting and correcting floating-point errors in matrix multiplication. On the theoretical side, we have detailed both methods, their variants, their common characteristics and their differences. On the practical side, we have implemented two variants for error correction in each method, one based on solving a small linear system, and one based on recomputing only corrupted elements, using coordinate checksumming to locate them. An extensive experimental comparison reveals similar execution times for the core of each method, but ABFT requires to embed the checksum in the user data in order to benefit from the high performance kernel implementation, while RC does not. Also, the flexibility of RC becomes very important when error rates are high, because $\mathrm{RC}$ can adapt a posteriori to the number of errors encountered within each particular execution. On the contrary, ABFT protection is constructed in a rigid way, with a fixed number of checksums which will rarely match the exact number of errors striking in a given run. This represents an acceptable overhead when the number of errors is smaller than expected, but it leads to the failing of the method when the number of errors is higher than the maximum number of errors that can be tolerated. To summarize, we point out that RC can be extended to correct silent errors in addition to detecting them, in a flexible and adaptive way, and without the burden of the extra memory allocation required by ABFT.

Future work will be devoted to extending the approaches to other linear algebra kernels, and to protect from memory corruptions in addition to floating-point errors. 


\section{References}

[1] Argyrides, C., Lisboa, C.A.L., Pradhan, D.K., Carro, L.: A fast error correction technique for matrix multiplication algorithms. In: 2009 15th IEEE International On-Line Testing Symposium. pp. 133-137 (June 2009). https://doi.org/10.1109/IOLTS.2009.5195995

[2] Benoit, A., Cavelan, A., Cappello, F., Raghavan, P., Robert, Y., Sun, H.: Coping with silent and fail-stop errors at scale by combining replication and checkpointing. J. Parallel Distributed Comput. 122, 209-225 (2018)

[3] Bosilca, G., Delmas, R., Dongarra, J., Langou, J.: Algorithmbased fault tolerance applied to high performance computing. J. Parallel Distrib. Comput. 69, 410-416 (2009). https://doi.org/doi:10.1016/j.jpdc.2008.12.002

[4] Bouteiller, A., Herault, T., Bosilca, G., Du, P., Dongarra, J.J.: Algorithm-based fault tolerance for dense matrix factorizations, multiple failures and accuracy. ACM Trans. Parallel Comput. 1(2), 10:110:28 (2015)

[5] Cappello, F., Geist, A., Gropp, W., Kale, S., Kramer, B., Snir, M.: Toward Exascale Resilience: 2014 update. Supercomputing frontiers and innovations $\mathbf{1}(1)$ (2014)

[6] Chen, Z., Dongarra, J.: Algorithm-based checkpoint-free fault tolerance for parallel matrix multiplications on volatile resources. In: Proceedings of the 20th IEEE International Parallel \& Distributed Processing Symposium, Rhodes Island, Greece, April 25-29 (2006)

[7] Chen, Z., Dongarra, J.J.: Condition numbers of gaussian random matrices. SIAM Journal on Matrix Analysis and Applications 27(3), 603620 (2005). https://doi.org/10.1137/040616413, https://doi .org/10. $1137 / 040616413$

[8] Chen, Z., Dongarra, J.J.: Numerically stable real number codes based on random matrices. In: Computational Science - ICCS 2005. ICCS 2005. Lecture Notes in Computer Science, vol 3514. Springer, Berlin, Heidelberg (2005)

[9] Fasi, M., Langou, J., Robert, Y., Uçar, B.: A backward/forward recovery approach for the preconditioned conjugate gradient method. J. Computational Science 17, 522-534 (2016)

[10] Gunnels, J., Katz, D., Quintana-Ortí, E., Van de Geijn, R.: Faulttolerant high-performance matrix multiplication: Theory and practice. 
In: Young, D., Young, D. (eds.) Proceedings of the International Conference on Dependable Systems and Networks. pp. 47-56 (Dec 2001). https://doi.org/10.1109/DSN.2001.941390

[11] Herault, T., Robert, Y. (eds.): Fault-Tolerance Techniques for HighPerformance Computing. Computer Communications and Networks, Springer Verlag (2015)

[12] Higham, N.J., Mary, T.: A new approach to probabilistic rounding error analysis. SIAM Journal on Scientific Computing 41(5), A2815-A2835 (2019). https://doi.org/10.1137/18M1226312

[13] Huang, K., Abraham, J.: Algorithm-based fault tolerance for matrix operations. IEEE Trans. on Comp. (Spec. Issue Reliable \& FaultTolerant Comp.) 33, 518-528 (1984)

[14] Lyons, R.E., Vanderkulk, W.: The use of triple-modular redundancy to improve computer reliability. IBM J. Res. Dev. 6(2), 200-209 (1962)

[15] Ni, X., Meneses, E., Jain, N., Kalé, L.V.: ACR: Automatic Checkpoint/Restart for Soft and Hard Error Protection. In: SC. ACM (2013)

[16] Plank, J.S.: A tutorial on Reed-Solomon coding for fault-tolerance in RAID-like systems. Software - Practice \& Experience 27(9), 995-1012 (1997)

[17] Prata, P., Silva, J.G.: Algorithm based fault tolerance versus result-checking for matrix computations. In: Digest of Papers. Twenty-Ninth Annual International Symposium on FaultTolerant Computing (Cat. No.99CB36352). pp. 4-11 (Jun 1999). https://doi.org/10.1109/FTCS.1999.781028

[18] Prata, P., Silva, J.G.: Fault-detection by result-checking for the eigenproblem. In: Hlavička, J., Maehle, E., Pataricza, A. (eds.) Dependable Computing - EDCC-3. pp. 419-436. Springer Berlin Heidelberg, Berlin, Heidelberg (1999)

[19] Reed, I.S., Solomon, G.: Polynomial codes over certain finite fields. Journal of the Society for Industrial and Applied Mathematics 8(2), 300-304 (1960). https://doi.org/10.1137/0108018

[20] Roy-Chowdhury, A., Banerjee, P.: Algorithm-based fault location and recovery for matrix computations on muitiprocessor systems. IEEE Transactions on Computers 45(11) (1996)

[21] Smith, T.M., van de Geijn, R.A., Smelyanskiy, M., Quintana-Ortí, E.S.: Towards ABFT for BLIS GEMM. Tech. Rep. 76, FLAME Working Note (June 2015) 
Detection and correction of floating-point errors in matrix-matrix multiplication 22

[22] Wu, P., Guan, Q., DeBardeleben, N., Blanchard, S., Tao, D., Liang, X., Chen, J., Chen, Z.: Towards practical algorithm based fault tolerance in dense linear algebra. In: Proceedings of the 25th ACM International Symposium on High-Performance Parallel and Distributed Computing. p. 31-42. HPDC '16, Association for Computing Machinery, New York, NY, USA (2016). https://doi.org/10.1145/2907294.2907315, https:// doi.org/10.1145/2907294.2907315 


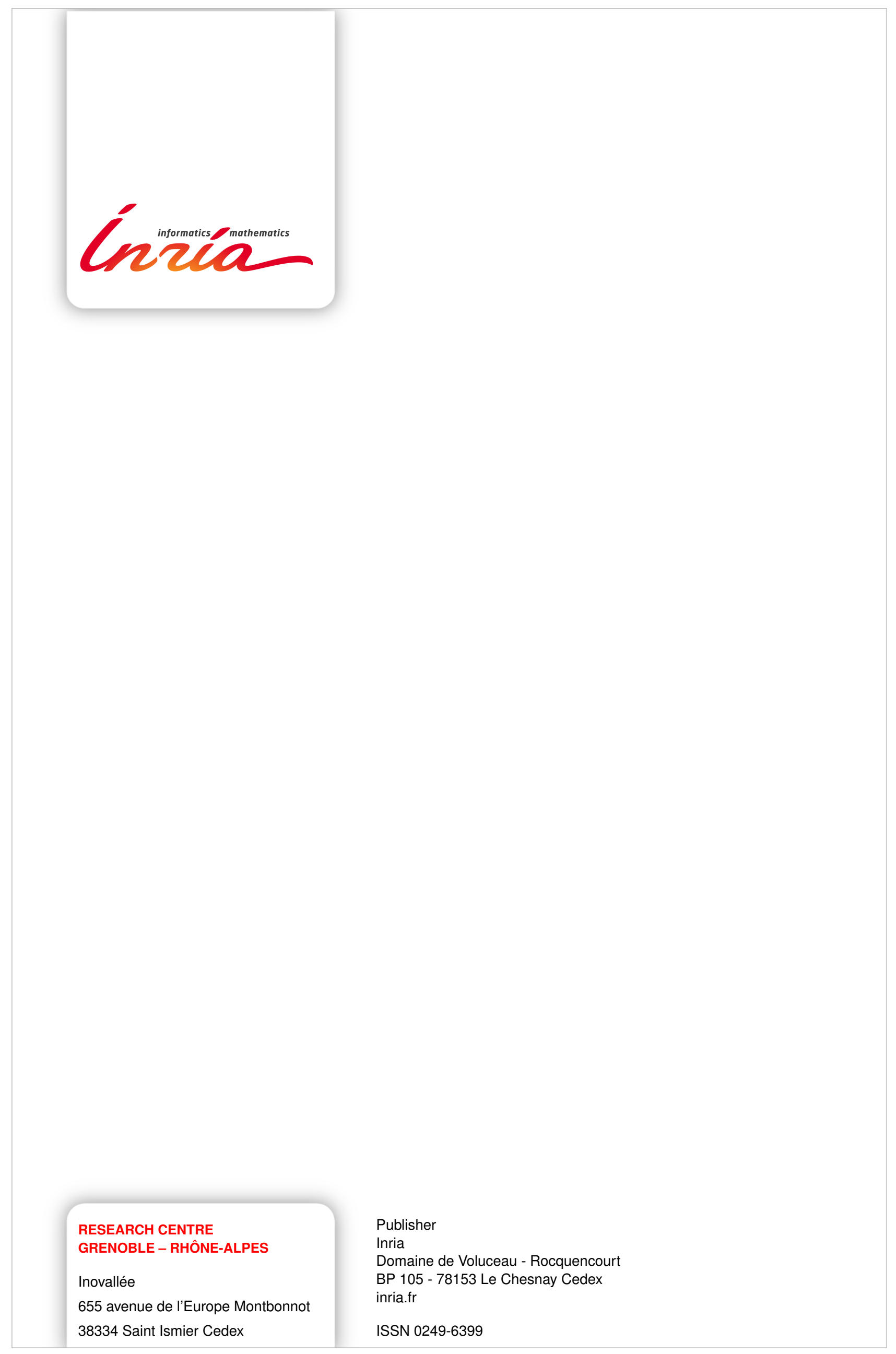

